\title{
DEGENERATE DEFORMATIONS AND UNIQUENESS IN HIGHLY ELASTIC NETWORKS
}

\author{
BY \\ W. A. GREEN (Nottingham University, U.K.) \\ AND \\ JINGYU SHI (University of Queensland, Australia)
}

\begin{abstract}
This work deals with the continuum theory for plane deformations of a network formed of two families of highly elastic cords, under the assumption of no resistance to shearing. Following Pipkin [3] it is shown that there exists a collapse mode of deformation in which a finite region of the network collapses onto a single curve and examples are exhibited which correspond to a universal deformation and to a universal state of tension. It is further shown that the assumption that the cords can withstand no compression leads to the existence of half-slack and fully-slack regions, as defined by Pipkin [5]. The most general deformation associated with a half-slack region is determined. A variational principle is established for the general boundary value problem and it is shown that, for strain-energy functions which are quadratic in the stretches of the cords, this leads to a minimum principle and a generalized uniqueness theorem. A stability and uniqueness theorem is derived for the materials with a more general strain energy function.
\end{abstract}

1. Introduction. The continuum theory for plane deformations of networks formed by two families of continuously distributed inextensible cords was first formulated by Rivlin [1]. Rivlin's theory, which assumes no shearing resistance between the cords, was subsequently applied by Rogers and Pipkin [2] to treat problems of inextensible networks with holes. An extension of Rivlin's model to include shear effects was proposed by Pipkin [3, 4], who also discussed some of the singularities that may occur in the solutions. A later paper by Pipkin [5] deals with a modification of Rivlin's theory so that the cords may shorten but not lengthen and may transmit tension but not compression. Pipkin [5, 6] also treats problems of existence and uniqueness of solutions in this modified theory, whilst Pipkin and Rogers [7] present a further extension to account for wrinkling of the network.

Amongst the degenerate or singular solutions that can arise in these theories, Pipkin [3] has identified a collapse mode of deformation in which a finite region of the 
undeformed network maps onto a singular curve in the deformed state. This collapse mode is a consequence of the inability of the network to resist shear. The inability of the cords to withstand compression can lead to the existence of half-slack regions, in which cords of one family are free of stress whilst those of the other are in tension; and of fully-slack regions, in which cords of both families are stress free [5]. As a result of these investigations there now exists a well-developed theory of the behaviour of these inextensible networks.

No corresponding body of results exists for networks formed by two families of extensible elastic cords. The continuum theory for the small strain linear elastic response has been formulated by Genensky and Rivlin [8] who have obtained solutions to the displacement boundary-value problem, the traction boundary-value problem and the mixed boundary-value problem. We have recently formulated a continuum theory for the plane deformations of a network formed from two families of highly elastic cords [9]. In that theory we make the assumption that the network exhibits no resistance to shear and that the cords cannot transmit compression. Some of the deformations and stress states that may exist in this network are exhibited in our paper [9] and additional examples are to be found in the thesis by Shi [10]. Here we are concerned with the question of uniqueness of solutions to boundary-value problems in the network and with degenerate modes of deformation. In Sec. 2 of this paper we derive the equilibrium equations under no body forces for a network formed of two families of elastic cords, with every member of one family initially orthogonal to every member of the other family and assuming no slipping at the points of intersection. We further assume that the network exhibits no resistance to shear and in consequence there exists the possibility of deformations involving a collapse region. These collapse regions are investigated in Sec. 3. Following Pipkin [5], we postulate that the cords have no bending stiffness. If then there exists a deformed region in which the cords of one family appear to be shorter than their natural lengths we interpret this as being brought about by an out-of-plane wrinkling of the network and those cords carry no load. This gives rise to the existence of deformations involving half-slack regions and these are examined in Sec. 4. Finally, in Sec. 5 we introduce a functional associated with the general boundary-value problem and we show that the governing equations follow from the requirement that this functional be stationary with respect to its independent variables. This functional involves the strain-energy functions of the two families of elastic cords. If these strain energies are assumed to be quadratic functions of the stretches then it can be shown that the stationary value of the functional is a local minimum. It is then possible to prove a generalized uniqueness theorem similar to that of Pipkin [5] for the inextensible network. The results derived by Hill [11] for finite strain deformations are used in Sec. 6 to discuss the uniqueness and stability for materials with a generalized strain energy function.

Our motivation for the present study lies partly in an attempt to model the behaviour of natural collageneous tissue such as skin and muscle. In this respect our assumptions of families of parallel elastic cords and of zero resistance to shearing lead to a highly idealized mathematical model which can at most be regarded as only a first step towards the attaining of this goal. Nevertheless the theory does have 
intrinsic interest and it can be regarded as a valid model for man-made materials woven from highly elastic cords.

2. General formulation. We consider a plane sheet of elastic material formed of two families of straight parallel elastic cords with the cords of one family being initially orthogonal to the cords of the other. Let $O x_{1} x_{2}$ be a system of plane Cartesian coordinates with axes parallel to the initial directions of the cords and consider a plane deformation in which a material point with initial coordinates $\left(X_{1}, X_{2}\right)$ relative to this system has coordinates $\left(x_{1}, x_{2}\right)$ in the deformed configuration, where

$$
x_{1}=x_{1}\left(X_{1}, X_{2}\right), \quad x_{2}=x_{2}\left(X_{1}, X_{2}\right) \text {. }
$$

We refer to the family of cords $X_{2}=$ constant as the $X_{1}$-cords and the family $X_{1}=$ constant as the $X_{2}$-cords. Then a line element along the $X_{1}$-cord at the point $\left(X_{1}, X_{2}\right)$ is deformed into a line element in the direction of the unit vector a given by

$$
\mathbf{a}=\left(x_{1,1} \mathbf{i}+x_{2,1} \mathbf{j}\right) / \lambda_{1},
$$

where $\mathbf{i}$ and $\mathbf{j}$ are unit vectors along $O x_{1}$ and $O x_{2}, x_{i, k}$ denotes differentiation with respect to $X_{k}$ and

$$
\lambda_{1}=\left(x_{1,1}^{2}+x_{2,1}^{2}\right)^{1 / 2}
$$

is the stretch of the line element. Letting $\varphi$ denote the angle between $\mathbf{a}$ and the unit vector $\mathbf{i}$ we have that

$$
x_{1,1}=\lambda_{1} \cos \varphi, \quad x_{2,1}=\lambda_{1} \sin \varphi,
$$

and the force $\widehat{\mathbf{T}}_{1}$ transmitted by the $X_{1}$-cord is given by

$$
\widehat{\mathbf{T}}_{1}=\widehat{T}_{1}\left(\lambda_{1}\right) \mathbf{a}=\widehat{T}_{1}\left(\lambda_{1}\right)(\cos \varphi \mathbf{i}+\sin \varphi \mathbf{j}) .
$$

Here the function $\widehat{T}_{1}\left(\lambda_{1}\right)$ is the response function associated with any member of the family of $X_{1}$-cords and is such that

$$
\begin{cases}\widehat{T}_{1}\left(\lambda_{1}\right)>0 & \text { for } \lambda_{1}>1, \\ \widehat{T}_{1}\left(\lambda_{1}\right)=0 & \text { for } \lambda_{1} \leq 1\end{cases}
$$

Similarly a line element in the direction of the $X_{2}$-cord at the point $\left(X_{1}, X_{2}\right)$ has the deformed direction of the unit vector $\mathbf{b}$ given by

$$
\mathbf{b}=\left(x_{1,2} \mathbf{i}+x_{2,2} \mathbf{j}\right) / \lambda_{2}=\cos \psi \mathbf{i}+\sin \psi \mathbf{j},
$$

where

$$
\begin{gathered}
\lambda_{2}=\left(x_{1,2}^{2}+x_{2,2}^{2}\right)^{1 / 2}, \\
x_{1,2}=\lambda_{2} \cos \psi, \quad x_{2,2}=\lambda_{2} \sin \psi,
\end{gathered}
$$

and $\psi$ is the angle between $\mathbf{b}$ and the $x_{1}$-axis. The force $\widehat{\mathbf{T}}_{2}$ transmitted by this $X_{2}$-cord is

$$
\widehat{\mathbf{T}}_{2}=\widehat{T}_{2}\left(\lambda_{2}\right) \mathbf{b}=\widehat{T}_{2}\left(\lambda_{2}\right)(\cos \psi \mathbf{i}+\sin \psi \mathbf{j}),
$$


where $\widehat{T}_{2}\left(\lambda_{2}\right)$ is the response function for any member of the family of $X_{2}$-cords and is such that

$$
\begin{cases}\widehat{T}_{2}\left(\lambda_{2}\right)>0 & \text { for } \lambda_{2}>1, \\ \widehat{T}_{2}\left(\lambda_{2}\right)=0 & \text { for } \lambda_{2} \leq 1 .\end{cases}
$$

Let $n_{1}$ be the number of cords of the $X_{1}$-family crossing unit length of any member of the $X_{2}$-family and let $n_{2}$ be the number of cords of the $X_{2}$-family crossing unit length of any member of the $X_{1}$-family. Then by considering the equilibrium of the quadrilateral formed by the deformation of an infinitesimal rectangle with sides $d X_{1}$ and $d X_{2}$ at $\left(X_{1}, X_{2}\right)$ we have that, in the absence of body forces, the forces $\widehat{\mathbf{T}}_{1}$ and $\widehat{\mathbf{T}}_{2}$ must satisfy the equilibrium equation

$$
\frac{\partial\left(n_{1} \widehat{\mathbf{T}}_{1}\right)}{\partial X_{1}}+\frac{\partial\left(n_{2} \widehat{\mathbf{T}}_{2}\right)}{\partial X_{2}}=\mathbf{0}
$$

Writing

$$
\mathbf{T}_{1}=n_{1} \widehat{\mathbf{T}}_{1}, \quad \mathbf{T}_{2}=n_{2} \widehat{\mathbf{T}}_{2},
$$

then $\mathbf{T}_{1}$ is the force per unit initial length acting across a line element of the $X_{2}$ cord through $\left(x_{1}, x_{2}\right)$ and $\mathbf{T}_{2}$ is the force per unit initial length acting across a line element of the $X_{1}$-cord through $\left(x_{1}, x_{2}\right)$. These are given, in terms of the response functions per unit initial length

$$
T_{1}\left(\lambda_{1}\right)=n_{1} \widehat{T}_{1}\left(\lambda_{1}\right), \quad T_{2}\left(\lambda_{2}\right)=n_{2} \widehat{T}_{2}\left(\lambda_{2}\right),
$$

by the expressions

$$
\begin{aligned}
& \mathbf{T}_{1}=T_{1}\left(\lambda_{1}\right)(\cos \varphi \mathbf{i}+\sin \varphi \mathbf{j})=T_{1}\left(\lambda_{1}\right)\left[\frac{x_{1,1} \mathbf{i}+x_{2,1} \mathbf{j}}{\lambda_{1}}\right], \\
& \mathbf{T}_{2}=T_{2}\left(\lambda_{2}\right)(\cos \psi \mathbf{i}+\sin \psi \mathbf{j})=T_{2}\left(\lambda_{2}\right)\left[\frac{x_{1,2} \mathbf{i}+x_{2,2} \mathbf{j}}{\lambda_{2}}\right],
\end{aligned}
$$

and the response functions $T_{1}\left(\lambda_{1}\right), T_{2}\left(\lambda_{2}\right)$ are such that

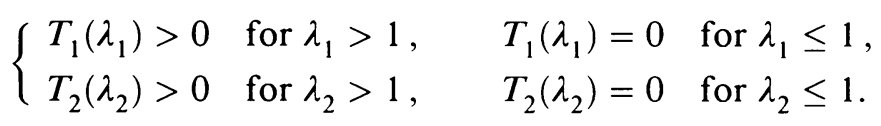

The equilibrium equations may then be written in component form as

$$
\begin{aligned}
& \frac{\partial}{\partial X_{1}}\left[T_{1}\left(\lambda_{1}\right) \cos \varphi\right]+\frac{\partial}{\partial X_{2}}\left[T_{2}\left(\lambda_{2}\right) \cos \psi\right]=0, \\
& \frac{\partial}{\partial X_{1}}\left[T_{1}\left(\lambda_{1}\right) \sin \varphi\right]+\frac{\partial}{\partial X_{2}}\left[T_{2}\left(\lambda_{2}\right) \sin \psi\right]=0,
\end{aligned}
$$

and equations (2.3) and (2.5) give rise to the compatibility relations

$$
\begin{aligned}
& \frac{\partial}{\partial X_{2}}\left(\lambda_{1} \cos \varphi\right)=\frac{\partial}{\partial X_{1}}\left(\lambda_{2} \cos \psi\right), \\
& \frac{\partial}{\partial X_{2}}\left(\lambda_{1} \sin \varphi\right)=\frac{\partial}{\partial X_{1}}\left(\lambda_{2} \sin \psi\right) .
\end{aligned}
$$


Equations (2.2)-(2.8) constitute the set of equations to be solved for the displacement functions $x_{1}\left(X_{1}, X_{2}\right), x_{2}\left(X_{1}, X_{2}\right)$ associated with the problem of prescribed displacement on the boundary of the network.

In order to deal with the traction boundary-value problem it is necessary to express the stretches $\lambda_{1}$ and $\lambda_{2}$ as functions of $T_{1}=\left|\mathbf{T}_{1}\right|$ and $T_{2}=\left|\mathbf{T}_{2}\right|$, respectively, and to solve equations (2.8) and (2.9) for $T_{1}, T_{2}, \varphi$, and $\psi$ subject to appropriate traction boundary conditions. Equations (2.3) and (2.5) may then be integrated to yield $x_{1}$ and $x_{2}$. It follows from equation (2.7) that in any region in which $T_{1}=0$ it is not possible to express $\lambda_{1}$ as a function of $T_{1}$ and that in any region in which $T_{2}=0$ the stretch $\lambda_{2}$ is not expressible as a function of $T_{2}$. Thus it is by no means evident that a solution will exist to any specified traction boundary-value problem and even if a solution exists it may well not be unique. The question of existence and uniqueness of the solutions forms part of this paper.

3. Collapsed regions. If there exists a deformation in which some region collapses into a single curve then the two cords through any point $\left(X_{1}, X_{2}\right)$ before the deformation must be parallel to each other after deformation so that either $\psi=\varphi$ or $\psi=\pi+\varphi$. Choosing first the case $\psi=\varphi$ then the equilibrium equations (2.8) become

$$
\begin{gathered}
\frac{\partial}{\partial X_{1}}\left(T_{1} \cos \varphi\right)+\frac{\partial}{\partial X_{2}}\left(T_{2} \cos \varphi\right)=0, \\
\frac{\partial}{\partial X_{1}}\left(T_{1} \sin \varphi\right)+\frac{\partial}{\partial X_{2}}\left(T_{2} \sin \varphi\right)=0,
\end{gathered}
$$

whilst the compatibility conditions $(2.9)$ reduce to

$$
\begin{aligned}
& \frac{\partial}{\partial X_{2}}\left(\lambda_{1} \cos \varphi\right)-\frac{\partial}{\partial X_{1}}\left(\lambda_{2} \cos \varphi\right)=0 \\
& \frac{\partial}{\partial X_{2}}\left(\lambda_{1} \sin \varphi\right)-\frac{\partial}{\partial X_{1}}\left(\lambda_{2} \sin \varphi\right)=0 .
\end{aligned}
$$

Expanding (3.1) and (3.2), and then rearranging them, we have

$$
\begin{aligned}
& \left(\frac{\partial T_{1}}{\partial X_{1}}+\frac{\partial T_{2}}{\partial X_{2}}\right) \cos \varphi-\left(T_{1} \frac{\partial \varphi}{\partial X_{1}}+T_{2} \frac{\partial \varphi}{\partial X_{2}}\right) \sin \varphi=0, \\
& \left(\frac{\partial T_{1}}{\partial X_{1}}+\frac{\partial T_{2}}{\partial X_{2}}\right) \sin \varphi+\left(T_{1} \frac{\partial \varphi}{\partial X_{1}}+T_{2} \frac{\partial \varphi}{\partial X_{2}}\right) \cos \varphi=0, \\
& \left(\frac{\partial \lambda_{1}}{\partial X_{2}}-\frac{\partial \lambda_{2}}{\partial X_{1}}\right) \cos \varphi-\left(\lambda_{1} \frac{\partial \varphi}{\partial X_{2}}-\lambda_{2} \frac{\partial \varphi}{\partial X_{1}}\right) \sin \varphi=0, \\
& \left(\frac{\partial \lambda_{1}}{\partial X_{2}}-\frac{\partial \lambda_{2}}{\partial X_{1}}\right) \sin \varphi+\left(\lambda_{1} \frac{\partial \varphi}{\partial X_{2}}-\lambda_{2} \frac{\partial \varphi}{\partial X_{1}}\right) \cos \varphi=0,
\end{aligned}
$$

and these may be rewritten as

$$
\begin{array}{ll}
\frac{\partial T_{1}}{\partial X_{1}}+\frac{\partial T_{2}}{\partial X_{2}}=0, & T_{1} \frac{\partial \varphi}{\partial X_{1}}+T_{2} \frac{\partial \varphi}{\partial X_{2}}=0, \\
\frac{\partial \lambda_{1}}{\partial X_{2}}-\frac{\partial \lambda_{2}}{\partial X_{1}}=0, & \lambda_{1} \frac{\partial \varphi}{\partial X_{2}}-\lambda_{2} \frac{\partial \varphi}{\partial X_{1}}=0 .
\end{array}
$$


If the collapsed region carries nonzero load then $\lambda_{1} T_{1}+\lambda_{2} T_{2} \neq 0$ and (3.5b) and (3.6b) have solution

$$
\frac{\partial \varphi}{\partial X_{1}}=\frac{\partial \varphi}{\partial X_{2}}=0
$$

or

$$
\varphi \equiv \text { constant }=\varphi_{0} .
$$

Equation (3.7) shows that the region collapses into a straight line. It follows that the tractions applied to the boundary of the collapsed region must everywhere be parallel to this line.

In a similar procedure, we find that if in some region $\psi=\pi+\varphi$, then the equations corresponding to (3.5a) and (3.6a) are

$$
\frac{\partial T_{1}}{\partial X_{1}}-\frac{\partial T_{2}}{\partial X_{2}}=0, \quad \frac{\partial \lambda_{1}}{\partial X_{2}}+\frac{\partial \lambda_{2}}{\partial X_{1}}=0,
$$

and that region also has to collapse into a straight line.

The tensions and stretches in the collapsed region can be determined from (3.5a) and (3.6a), or from (3.8), with the aid of the response functions

$$
T_{1}=T_{1}\left(\lambda_{1}\right), \quad T_{2}=T_{2}\left(\lambda_{2}\right),
$$

or their inverses

$$
\lambda_{1}=\lambda_{1}\left(T_{1}\right), \quad \lambda_{2}=\lambda_{2}\left(T_{2}\right) .
$$

The equation $(3.6 \mathrm{a})$ is satisfied by

$$
\lambda_{1}=\frac{\partial F}{\partial X_{1}}, \quad \lambda_{2}=\frac{\partial F}{\partial X_{2}},
$$

where $F\left(X_{1}, X_{2}\right)$ is an arbitrary function. Then

$$
\begin{array}{ll}
x_{1,1}=\lambda_{1} \cos \varphi_{0}=c_{0} \frac{\partial F}{\partial X_{1}}, & x_{1,2}=\lambda_{2} \cos \psi_{0}=c_{0} \frac{\partial F}{\partial X_{2}}, \\
x_{2,1}=\lambda_{1} \sin \varphi_{0}=s_{0} \frac{\partial F}{\partial X_{1}}, & x_{2,2}=\lambda_{2} \sin \psi_{0}=s_{0} \frac{\partial F}{\partial X_{2}},
\end{array}
$$

where $c_{0}=\cos \varphi_{0}=\cos \psi_{0}$ and $s_{0}=\sin \varphi_{0}=\sin \psi_{0}$ are constants. Equations (3.12) integrate to yield

$$
x_{1}=h+c_{0} F\left(X_{1}, X_{2}\right), \quad x_{2}=k+s_{0} F\left(X_{1}, X_{2}\right),
$$

where $h$ and $k$ are arbitrary constants. Equations (3.13) show that in the collapsed deformation the curve

$$
F\left(X_{1}, X_{2}\right)=C,
$$

where $C$ is a constant, collapses into the point $\left(h+c_{0} C, k+s_{0} C\right)$. As the parameter $C$ varies, the family of curves represented by equation (3.14) traces out the collapsed line

$$
s_{0}\left(x_{1}-h\right)-c_{0}\left(x_{2}-k\right)=0 .
$$


Substituting from (3.11) into the response functions (3.9), the equilibrium equation (3.5a) then becomes

$$
T_{1}^{\prime}\left(F_{, 1}\right) \frac{\partial^{2} F}{\partial X_{1}^{2}}+T_{2}^{\prime}\left(F_{, 2}\right) \frac{\partial^{2} F}{\partial X_{2}^{2}}=0,
$$

where the primes denote differentiation with respect to the argument. Equation (3.16) is a nonlinear second-order partial differential equation for the stretch potential function $F\left(X_{1}, X_{2}\right)$ and, in general, its solution will depend on the form of the response functions $T_{1}\left(\lambda_{1}\right)$ and $T_{2}\left(\lambda_{2}\right)$. A solution that satisfies this equation for all response functions is

$$
F\left(X_{1}, X_{2}\right)=A X_{1}+B X_{2}+D X_{1} X_{2}+E,
$$

where $A, B, D$, and $E$ are arbitrary constants.

For nonzero values of $D$ there is no loss of generality in choosing $A=B=E=0$ in equation (3.17). In that case, the one-parameter family of curves represented by equation (3.14) is a family of rectangular hyperbolae with the coordinate axes as asymptotes. The stretch $\lambda_{1}$ is a linear function of $X_{2}$ and the stretch $\lambda_{2}$ is a linear function of $X_{1}$, given by

$$
\lambda_{1}=\frac{\partial F}{\partial X_{1}}=D X_{2}, \quad \lambda_{2}=\frac{\partial F}{\partial X_{2}}=D X_{1} .
$$

Any portion of the network occupying the region $X_{1}>1 / D, X_{2}>1 / D$, before the deformation, collapses onto the line (3.15). The stress state at the point initially at $\left(X_{1}, X_{2}\right)$ is given by

$$
\begin{aligned}
& \mathbf{T}_{1}=T_{1}\left(D X_{2}\right)\left(\mathbf{i} \cos \varphi_{0}+\mathbf{j} \sin \varphi_{0}\right), \\
& \mathbf{T}_{2}=T_{2}\left(D X_{1}\right)\left(\mathbf{i} \cos \varphi_{0}+\mathbf{j} \sin \varphi_{0}\right) .
\end{aligned}
$$

If the boundary of the collapsed region prior to the deformation is a closed curve given parametrically in terms of arc length $S$ by the relations

$$
X_{1}=X_{1}(S), \quad X_{2}=X_{2}(S),
$$

then the deformation may be achieved by specifying that the boundary point $\left(X_{1}(S), X_{2}(S)\right)$ is displaced to the point $\left(h+c_{0} F(S), k+s_{0} F(S)\right)$ where

$$
F(S) \equiv F\left(X_{1}(S), X_{2}(S)\right) .
$$

The boundary traction $\mathbf{T}(S)$ required in order to maintain this deformation is

$$
\mathbf{T}(S)=\left\{T_{1}\left(D X_{2}(S)\right) \frac{d X_{1}}{d S}+T_{2}\left(D X_{1}(S)\right) \frac{d X_{2}}{d S}\right\}\left(\mathbf{i} \cos \varphi_{0}+\mathbf{j} \sin \varphi_{0}\right) .
$$

Thus, while the deformation is completely determined by the function $F\left(X_{1}, X_{2}\right)$, the state of stress and the tractions required to maintain the deformation depend on the particular response functions $T_{1}\left(\lambda_{1}\right)$ and $T_{2}\left(\lambda_{2}\right)$. This is therefore an example of a universal deformation.

A different universal deformation is obtained from the solution (3.17) by choosing $D=0$ so that

$$
F\left(X_{1}, X_{2}\right)=A X_{1}+B X_{2}+E
$$


The curves (3.14) are then a one-parameter family of parallel straight lines. The stretches are given by $\lambda_{1}=A, \lambda_{2}=B$ and, provided $A>1$ and $B>1$, the solution is valid and the deformed network is subjected to the homogeneous stress state

$$
\begin{aligned}
& \mathbf{T}_{1}=T_{1}(A)\left(\mathbf{i} \cos \varphi_{0}+\mathbf{j} \sin \varphi_{0}\right), \\
& \mathbf{T}_{2}=T_{2}(B)\left(\mathbf{i} \cos \varphi_{0}+\mathbf{j} \sin \varphi_{0}\right) .
\end{aligned}
$$

Returning to equations (3.5a) and (3.6a), we remark that the former is satisfied identically by introducing the stress function $G\left(X_{1}, X_{2}\right)$ such that

$$
T_{1}=\frac{\partial G}{\partial X_{2}}, \quad T_{2}=-\frac{\partial G}{\partial X_{1}} .
$$

Provided $\partial G / \partial X_{2}>0$ and $\partial G / \partial X_{1}<0$, the response functions (3.9) may be inverted to yield (3.10). Substituting these into (3.6a) using (3.20) gives the secondorder nonlinear partial differential equation for the stress function

$$
\lambda_{1}^{\prime}\left(G_{, 2}\right) \frac{\partial^{2} G}{\partial X_{2}^{2}}+\lambda_{2}^{\prime}\left(-G_{, 1}\right) \frac{\partial^{2} G}{\partial X_{1}^{2}}=0,
$$

where the primes denote differentiation with respect to the arguments. The solution of equation (3.21) depends on the form of the response functions (3.10) except for the case when

$$
G\left(X_{1}, X_{2}\right)=a X_{1}+b X_{2}+d X_{1} X_{2}+e,
$$

where $a, b, d$, and $e$ are arbitrary constants. For this solution the stress state is

$$
T_{1}=d X_{1}+b, \quad T_{2}=-\left(d X_{2}+a\right),
$$

which is valid provided $\left(d X_{1}+b\right)>0$ and $\left(d X_{2}+a\right)<0$. If the boundary of the collapsed region is the closed curve given parametrically by (3.19) then this deformation is maintained by the boundary traction

$$
\begin{aligned}
\mathbf{T}(S) & =\left[\left(d X_{1}+b\right) \frac{d X_{1}}{d S}-\left(d X_{2}+a\right) \frac{d X_{2}}{d S}\right]\left(\mathbf{i} \cos \varphi_{0}+\mathbf{j} \sin \varphi_{0}\right) \\
& =\frac{d}{d S}\left\{\frac{d\left(X_{1}^{2}-X_{2}^{2}\right)}{2}+b X_{1}-a X_{2}\right\}\left(\mathbf{i} \cos \varphi_{0}+\mathbf{j} \sin \varphi_{0}\right) .
\end{aligned}
$$

Equations (3.22) are valid for all materials of this class but the deformation depends on the form of the response functions (3.10). This is therefore an example of a universal state of stress.

4. Half-slack regions. We assume the half-slack region is such that $\lambda_{1}>1$ and $\lambda_{2} \leq 1$ so that $T_{2}=0$ and $T_{1}>0$ throughout the region. The equations of equilibrium (2.8) then simplify to

$$
\frac{\partial}{\partial X_{1}}\left(T_{1}\left(\lambda_{1}\right) \cos \varphi\right)=0, \quad \frac{\partial}{\partial X_{1}}\left(T_{1}\left(\lambda_{1}\right) \sin \varphi\right)=0,
$$

which integrate to yield

$$
T_{1}\left(\lambda_{1}\right) \cos \varphi=L\left(X_{2}\right), \quad T_{1}\left(\lambda_{1}\right) \sin \varphi=M\left(X_{2}\right),
$$


where $L\left(X_{2}\right)$ and $M\left(X_{2}\right)$ are arbitrary functions. Equations (4.1) imply that $\varphi=$ $\varphi\left(X_{2}\right)$ showing that each of the $X_{1}$-cords remains straight in the deformation. It also follows from (4.1) that whatever the form of the response function $T_{1}\left(\lambda_{1}\right)$ the stretch $\lambda_{1}$ is a function of $X_{2}$ only which, since $\lambda_{1}>1$, can be written as $\lambda_{1}=1+a^{2}\left(X_{2}\right)$, where $a\left(X_{2}\right)$ is an arbitrary function. The most general deformation associated with a half-slack region may then be expressed as

$$
\begin{aligned}
& x_{1}=X_{1}\left[1+a^{2}\left(X_{2}\right)\right] \cos \varphi\left(X_{2}\right)+f\left(X_{2}\right), \\
& x_{2}=X_{1}\left[1+a^{2}\left(X_{2}\right)\right] \sin \varphi\left(X_{2}\right)+g\left(X_{2}\right),
\end{aligned}
$$

where $f\left(X_{2}\right)$ and $g\left(X_{2}\right)$ are arbitrary functions. Eliminating $X_{1}$ between the two equations (4.2) leads to the relation

$$
x_{1} \sin \varphi\left(X_{2}\right)-x_{2} \cos \varphi\left(X_{2}\right)=h\left(X_{2}\right),
$$

where $h\left(X_{2}\right)=f\left(X_{2}\right) \sin \varphi\left(X_{2}\right)-g\left(X_{2}\right) \cos \varphi\left(X_{2}\right)$. Equation (4.3) represents a oneparameter family of straight lines each member of which corresponds to one of the $X_{1}$-cords. This family will envelope a curve whose equation in the deformed configuration is obtained by eliminating the parameter $X_{2}$ between equation (4.3) and the equation derived from it by differentiating with respect to $X_{2}$

$$
x_{1} \cos \varphi\left(X_{2}\right)+x_{2} \sin \varphi\left(X_{2}\right)=h^{\prime}\left(X_{2}\right) / \varphi^{\prime}\left(X_{2}\right) .
$$

Examples of half-slack regions and their associated envelopes are presented in our earlier paper [9].

The deformation given by equations (4.2) is valid for values of $X_{1}, X_{2}$ such that the stretch $\lambda_{2}$ is less than or equal to unity and the stress $T_{2}$ is then zero. The boundaries of the half-slack region therefore consist of portions of the straight lines formed by the $X_{1}$-cords and the curve or curves on which $\lambda_{2}=1$. We point out here that the straight line segments formed by the $X_{1}$-cords cannot form the boundary between a half-slack region and a noncollapsed fully stretched region, since in the former region $T_{2}=0$ and in the latter region $T_{2}>0$ and is not parallel to the boundary. We see from equation (3.22), however, that the $X_{1}$-cord can form the boundary between the half-slack region and a collapsed region since, by an appropriate choice of the constants $d$ and $a$, it is possible to make $T_{2}=0$ in equation (3.22) for any given constant value of $X_{2}$.

5. Variational formulation of the governing equations. In this section we derive the variational formulation of the boundary-value problem. To do this we put $\varphi=\varphi_{1}$, $\psi=\varphi_{2}$ and define a functional $\Pi\left(x_{\alpha}, \lambda_{\alpha}, \varphi_{\alpha}, T_{\alpha \beta}\right)$ by the expression

$$
\begin{aligned}
\Pi=\iint_{A}\left\{\widetilde{W}_{1}\left(\lambda_{1}\right)+\widetilde{W}_{2}\left(\lambda_{2}\right)\right. \\
\left.\quad+\sum_{\alpha=1}^{2}\left[T_{\alpha 1}\left(x_{1, \alpha}-\lambda_{\alpha} \cos \varphi_{\alpha}\right)+T_{\alpha 2}\left(x_{2, \alpha}-\lambda_{\alpha} \sin \varphi_{\alpha}\right)\right]\right\} d A \\
\quad-\int_{S_{T}}\left\{\bar{T}_{1} x_{1}+\bar{T}_{2} x_{2}\right\} d S .
\end{aligned}
$$


Here $x_{\alpha}, \lambda_{\alpha}, \varphi_{\alpha}$, and $T_{\alpha \beta}(\alpha, \beta=1,2)$ are arbitrary functions of position $\left(X_{1}, X_{2}\right) . \bar{T}_{1}$ and $\bar{T}_{2}$ are the traction components specified on the portion $S_{T}$ of the boundary curve $S$ of the area $A$, and $S_{u}=\left(S-S_{T}\right)$ is the portion of the boundary curve on which $x_{\alpha}=\bar{x}_{\alpha}(\alpha=1,2)$ is prescribed. Note that $A$ and $S$ refer to the undeformed configuration. The functions $\widetilde{W}_{1}\left(\lambda_{1}\right)$ and $\widetilde{W}_{2}\left(\lambda_{2}\right)$ are defined as

$$
\begin{aligned}
& \widetilde{W}_{1}\left(\lambda_{1}\right)= \begin{cases}\frac{W_{1}\left(\lambda_{1}\right)}{2}\left[1+\frac{\lambda_{1}-1}{\left|\lambda_{1}-1\right|}\right] & \text { if } \lambda_{1} \neq 1, \\
0 & \text { if } \lambda_{1}=1,\end{cases} \\
& \widetilde{W}_{2}\left(\lambda_{2}\right)= \begin{cases}\frac{W_{2}\left(\lambda_{2}\right)}{2}\left[1+\frac{\lambda_{2}-1}{\left|\lambda_{2}-1\right|}\right] & \text { if } \lambda_{2} \neq 1, \\
0 & \text { if } \lambda_{2}=1,\end{cases}
\end{aligned}
$$

where $W_{1}\left(\lambda_{1}\right)$ and $W_{2}\left(\lambda_{2}\right)$ are positive and increase as their arguments increase in the region $\lambda_{\alpha}>1 \quad(\alpha=1,2)$.

We allow arbitrary variations in $\lambda_{\alpha}, \varphi_{\alpha}, x_{\alpha}, T_{\alpha 1}$, and $T_{\alpha 2}(\alpha=1,2)$ and we require that $\Pi$ be stationary for such variations, i.e.,

$$
\begin{aligned}
& \delta \Pi=0=\iint_{A}\left\{\frac{d \widetilde{W}_{1}}{d \lambda_{1}} \delta \lambda_{1}+\frac{d \widetilde{W}_{2}}{d \lambda_{2}} \delta \lambda_{2}\right. \\
& +\sum_{\alpha=1}^{2}\left[\delta T_{\alpha 1}\left(x_{1, \alpha}-\lambda_{\alpha} \cos \varphi_{\alpha}\right)+\delta T_{\alpha 2}\left(x_{2, \alpha}-\lambda_{\alpha} \sin \varphi_{\alpha}\right)\right. \\
& +T_{\alpha 1}\left(\delta x_{1, \alpha}-\delta \lambda_{\alpha} \cos \varphi_{\alpha}+\lambda_{\alpha} \sin \varphi_{\alpha} \delta \varphi_{\alpha}\right) \\
& \left.\left.+T_{\alpha 2}\left(\delta x_{2, \alpha}-\delta \lambda_{\alpha} \sin \varphi_{\alpha}-\lambda_{\alpha} \cos \varphi_{\alpha} \delta \varphi_{\alpha}\right)\right]\right\} d A \\
& -\int_{S_{T}}\left\{\bar{T}_{1} \delta x_{1}+\bar{T}_{2} \delta x_{2}\right\} d S \\
& =\iint_{A} \sum_{\alpha=1}^{2}\left[\left(\frac{d \widetilde{W}_{\alpha}}{d \lambda_{\alpha}}-T_{\alpha 1} \cos \varphi_{\alpha}-T_{\alpha 2} \sin \varphi_{\alpha}\right) \delta \lambda_{\alpha}\right. \\
& +\left(x_{1, \alpha}-\lambda_{\alpha} \cos \varphi_{\alpha}\right) \delta T_{\alpha 1}+\left(x_{2, \alpha}-\lambda_{\alpha} \sin \varphi_{\alpha}\right) \delta T_{\alpha 2} \\
& \left.+\left(T_{\alpha 1} \sin \varphi_{\alpha}-T_{\alpha 2} \cos \varphi_{\alpha}\right) \lambda_{\alpha} \delta \varphi_{\alpha}+T_{\alpha 1} \delta x_{1, \alpha}+T_{\alpha 2} \delta x_{2, \alpha}\right] d A \\
& -\int_{S_{T}}\left[\bar{T}_{1} \delta x_{1}+\bar{T}_{2} \delta x_{2}\right] d S .
\end{aligned}
$$

Since all variations are arbitrary we obtain the following results:

$$
\begin{gathered}
\frac{d \widetilde{W}_{\alpha}}{d \lambda_{\alpha}}-T_{\alpha 1} \cos \varphi_{\alpha \alpha}-T_{\alpha 2} \sin \varphi_{\alpha}=0, \quad(\alpha=1,2), \\
\left\{\begin{array}{l}
x_{1, \alpha}-\lambda_{\alpha} \cos \varphi_{\alpha}=0, \\
x_{2, \alpha}-\lambda_{\alpha} \sin \varphi_{\alpha}=0,
\end{array} \quad(\alpha=1,2),\right. \\
T_{\alpha 1} \sin \varphi_{\alpha}-T_{\alpha 2} \cos \varphi_{\alpha}=0, \quad(\alpha=1,2),
\end{gathered}
$$


and we are left with

$$
\delta \Pi=\iint_{A} \sum_{\alpha=1}^{2}\left(T_{\alpha 1} \delta x_{1, \alpha}+T_{\alpha 2} \delta x_{2, \alpha}\right) d A-\int_{S_{T}}\left(\bar{T}_{1} \delta x_{1}+\bar{T}_{2} \delta x_{2}\right) d S=0 .
$$

If the arbitrary variations $\delta x_{1}$ and $\delta x_{2}$ are such that $\delta x_{1}=\delta x_{2}=0$ on $S_{u}$, then by using Gauss's theorem,

$$
\begin{aligned}
\delta \Pi= & \int_{S_{T}}\left[\left(\sum_{\alpha=1}^{2} T_{\alpha 1} N_{\alpha}-\bar{T}_{1}\right) \delta x_{1}-\left(\sum_{\alpha=1}^{2} T_{\alpha 2} N_{\alpha}-\bar{T}_{2}\right) \delta x_{2}\right] d S \\
& -\iint_{A} \sum_{\alpha=1}^{2}\left(\frac{\partial T_{\alpha 1}}{\partial X_{\alpha}} \delta x_{1}+\frac{\partial T_{\alpha 2}}{\partial X_{\alpha}} \delta x_{2}\right) d A=0,
\end{aligned}
$$

where $\left(N_{1}, N_{2}\right)$ is the unit normal vector of $S$. This gives

$$
\sum_{\alpha=1}^{2} \frac{\partial T_{\alpha 1}}{\partial X_{\alpha}}=0, \quad \sum_{\alpha=1}^{2} \frac{\partial T_{\alpha 2}}{\partial X_{\alpha}}=0 \quad \text { in } A
$$

and

$$
\bar{T}_{1}=\sum_{\alpha=1}^{2} T_{\alpha 1} N_{\alpha}, \quad \bar{T}_{2}=\sum_{\alpha=1}^{2} T_{\alpha 2} N_{\alpha} \text { on } S_{T} .
$$

The equations (5.4) are the kinematic relations (2.3) and (2.5), which give the compatibility conditions (2.9). The equations (5.5) are satisfied by introducing functions $T_{1}\left(X_{1}, X_{2}\right)$ and $T_{2}\left(X_{1}, X_{2}\right)$ and writing

$$
T_{\alpha 1}=T_{\alpha} \cos \varphi_{\alpha}, \quad T_{\alpha 2}=T_{\alpha} \sin \varphi_{\alpha}, \quad(\alpha=1,2) .
$$

Then the equations (5.3) give

$$
T_{\alpha}=\frac{d \widetilde{W}_{\alpha}}{d \lambda_{\alpha}}=\frac{1}{2} \frac{d W_{\alpha}}{d \lambda_{\alpha}}\left[1+\frac{\lambda_{\alpha}-1}{\left|\lambda_{\alpha}-1\right|}\right]=\left\{\begin{array}{ll}
\frac{d W_{\alpha}}{d \lambda_{\alpha}}, & \left(\lambda_{\alpha}>1\right), \\
0, & \left(\lambda_{\alpha} \leq 1\right),
\end{array} \quad(\alpha=1,2) .\right.
$$

This implies that $T_{\alpha}(\alpha=1,2)$ are tensions along cord lines and $\left(\cos \varphi_{\alpha}, \sin \varphi_{\alpha}\right)$ are their directions. Therefore the equations (5.3) or (5.9) are stretch-tension relations. Furthermore, $T_{\alpha \beta}(\alpha, \beta=1,2)$ are components of the Piola-Kirchhoff (or nominal) stress tensor. With the aid of equations (5.8) it may be seen that equations (5.6) are then the equilibrium equations (2.8), and that equations (5.7) are the traction boundary conditions. We thus see that the requirement that the first-order variation of the functional (5.1) should be zero is fully equivalent to the governing equations and traction boundary conditions which we have formulated in Sec. 2.

Now we investigate the extremum principles. Let $\lambda_{\alpha}^{*}, \varphi_{\alpha}^{*}, x_{\alpha}^{*}, T_{\alpha 1}^{*}$, and $T_{\alpha 2}^{*}$ $(\alpha=1,2)$ be a solution satisfying the prescribed boundary conditions. Let $\hat{\lambda}_{\alpha}, \hat{\varphi}_{\alpha}$, $\hat{x}_{\alpha}, \widehat{T}_{\alpha 1}$, and $\widehat{T}_{\alpha 2}$ satisfy the equations (5.3), (5.4), and (5.5), but not necessarily 
(5.6) and (5.7). Also $\hat{x}_{\alpha}$ must satisfy the boundary conditions on $S_{u}$. Then

$$
\begin{aligned}
\widehat{\Pi}-\Pi^{*}= & \iint_{A}\left[\widetilde{W}_{1}\left(\hat{\lambda}_{1}\right)+\widetilde{W}_{2}\left(\hat{\lambda}_{2}\right)-\widetilde{W}_{1}\left(\lambda_{1}^{*}\right)-\widetilde{W}_{2}\left(\lambda_{2}^{*}\right)\right] d A \\
& -\int_{S_{T}}\left[\widetilde{T}_{1}\left(\hat{x}_{1}-x_{1}^{*}\right)+\widetilde{T}_{2}\left(\hat{x}_{2}-x_{2}^{*}\right)\right] d S \\
= & \iint_{A}\left[\widetilde{W}_{1}\left(\hat{\lambda}_{1}\right)+\widetilde{W}_{2}\left(\hat{\lambda}_{2}\right)-\widetilde{W}_{1}\left(\lambda_{1}^{*}\right)-\widetilde{W}_{2}\left(\lambda_{2}^{*}\right)\right] d A \\
& -\iint_{A}\left[T_{1}^{*}\left(\hat{\lambda}_{1}^{*}-\lambda_{1}^{*}\right)+T_{2}^{*}\left(\hat{\lambda}_{2}^{*}-\lambda_{2}^{*}\right)\right] d A .
\end{aligned}
$$

Here $T_{\alpha}^{*}(\alpha=1,2)$ are tensions arising in the solution and the quantities $\hat{\lambda}_{1}^{*}, \hat{\lambda}_{2}^{*}$ are defined to be

$$
\hat{\lambda}_{1}^{*}=\hat{\lambda}_{1} \cos \left(\varphi_{1}^{*}-\hat{\varphi}_{1}\right), \quad \hat{\lambda}_{2}^{*}=\hat{\lambda}_{2} \cos \left(\varphi_{2}^{*}-\hat{\varphi}_{2}\right) .
$$

We now postulate that the strain-energy functions $W_{1}\left(\lambda_{1}\right), W_{2}\left(\lambda_{2}\right)$, associated with each family of cords, are quadratic functions of the stretches, given by

$$
W_{1}\left(\lambda_{1}\right)=\frac{1}{2} E_{1}\left(\lambda_{1}-1\right)^{2}, \quad W_{2}\left(\lambda_{2}\right)=\frac{1}{2} E_{2}\left(\lambda_{2}-1\right)^{2} .
$$

Then

$$
T_{1}^{*}=\left\{\begin{array}{ll}
E_{1}\left(\lambda_{1}^{*}-1\right), & \lambda_{1}^{*}>1, \\
0, & \lambda_{1}^{*} \leq 1,
\end{array} \quad T_{2}^{*}= \begin{cases}E_{2}\left(\lambda_{2}^{*}-1\right), & \lambda_{2}^{*}>1, \\
0, & \lambda_{2}^{*} \leq 1,\end{cases}\right.
$$

and we can write

$$
\begin{aligned}
T_{1}^{*}\left(\hat{\lambda}_{1}^{*}-\lambda_{1}^{*}\right) & =\frac{1}{2}\left(1+\frac{\lambda_{1}^{*}-1}{\left|\lambda_{1}^{*}-1\right|}\right)\left[E_{1}\left(\lambda_{1}^{*}-1\right)\left(\hat{\lambda}_{1}^{*}-1\right)-E_{1}\left(\lambda_{1}^{*}-1\right)^{2}\right] \\
& =\frac{1}{2}\left(1+\frac{\lambda_{1}^{*}-1}{\left|\lambda_{1}^{*}-1\right|}\right)\left[\left(\hat{\lambda}_{1}^{*}-1\right)^{2}-\left(\lambda_{1}^{*}-1\right)^{2}-\left(\lambda_{1}^{*}-\hat{\lambda}_{1}^{*}\right)^{2}\right] \frac{E_{1}}{2} .
\end{aligned}
$$

In the same way we have that

$$
T_{2}^{*}\left(\hat{\lambda}_{2}^{*}-\lambda_{2}^{*}\right)=\frac{1}{2}\left(1+\frac{\lambda_{2}^{*}-1}{\left|\lambda_{2}^{*}-1\right|}\right)\left[\left(\hat{\lambda}_{2}^{*}-1\right)^{2}-\left(\lambda_{2}^{*}-1\right)^{2}-\left(\lambda_{2}^{*}-\hat{\lambda}_{2}^{*}\right)^{2}\right] \frac{E_{2}}{2} .
$$

Substituting these expressions into the last integral in equation (5.10) and expressing the first integral in terms of (5.2) and (5.12) gives

$$
\begin{aligned}
& \widehat{\Pi}-\Pi^{*}= \frac{E_{1}}{2} \iint_{A}\left\{\frac{1}{2}\left(1+\frac{\lambda_{1}^{*}-1}{\left|\lambda_{1}^{*}-1\right|}\right)\left[\left(\lambda_{1}^{*}-\hat{\lambda}_{1}^{*}\right)^{2}-\left(1-\hat{\lambda}_{1}^{*}\right)^{2}\right]\right. \\
&\left.+\frac{1}{2}\left(1+\frac{\hat{\lambda}_{1}-1}{\left|\hat{\lambda}_{1}-1\right|}\right)\left(\hat{\lambda}_{1}-1\right)^{2}\right\} d A \\
&+ \frac{E_{2}}{2} \iint_{A}\left\{\frac{1}{2}\left(1+\frac{\lambda_{2}^{*}-1}{\left|\lambda_{2}^{*}-1\right|}\right)\left[\left(\lambda_{2}^{*}-\hat{\lambda}_{2}^{*}\right)^{2}-\left(1-\hat{\lambda}_{2}^{*}\right)^{2}\right]\right. \\
&\left.+\frac{1}{2}\left(1+\frac{\hat{\lambda}_{2}-1}{\left|\hat{\lambda}_{2}-1\right|}\right)\left(\hat{\lambda}_{2}-1\right)^{2}\right\} d A
\end{aligned}
$$


Consider the first of the two integrals in equation (5.13). In any subregion $A_{1}$ of $A$ in which $\hat{\lambda}_{1} \leq 1$ the last term of this integral is zero. Moreover, since $\hat{\lambda}_{1}^{*} \leq \hat{\lambda}_{1}$ from the definition (5.11), it follows that in $A_{1}, \hat{\lambda}_{1}^{*} \leq 1$ also. The first term of this integral vanishes in any part of the region $A_{1}$ in which $\lambda_{1}^{*}<1$ and in the remainder of $A_{1}$ this term becomes $\left[\left(\lambda_{1}^{*}-\hat{\lambda}_{1}^{*}\right)^{2}-\left(1-\hat{\lambda}_{1}^{*}\right)^{2}\right]$, which is strictly positive since $\lambda_{1}^{*}>1$ and $\hat{\lambda}_{1}^{*}<1$ in this region. It follows that the contribution to the integral from the region $A_{1}$ is nonnegative. In the remaining region $\left(A-A_{1}\right)$ the stretch $\hat{\lambda}_{1}>1$, and the last term in the integral is $\left(\hat{\lambda}_{1}-1\right)^{2}$, which is strictly positive. Further, if there exists a subregion of $\left(A-A_{1}\right)$ in which $\lambda_{1}^{*} \leq 1$, the first term of the integral vanishes and the contribution from this region is strictly positive. In the remainder of the region $\left(A-A_{1}\right)$ we have that $\lambda_{1}^{*}>1$ and $\hat{\lambda}_{1}>1$ and the integrand becomes

$$
\left(\lambda_{1}^{*}-\hat{\lambda}_{1}^{*}\right)^{2}+\left(\hat{\lambda}_{1}-1\right)^{2}-\left(\hat{\lambda}_{1}^{*}-1\right)^{2}
$$

in which $\hat{\lambda}_{1}^{*} \leq \hat{\lambda}_{1}$. Two possibilities arise: (i) if $\hat{\lambda}_{1}^{*} \geq 1$ then the second term is greater than or equal to the third (with equality iff $\hat{\lambda}_{1}^{*}=\hat{\lambda}_{1}$ ) and the integrand is nonnegative; (ii) if $\hat{\lambda}_{1}^{*}<1$ then the first term is strictly greater than the third term and the integrand is positive.

Summing up, we have that the first of the two integrals in (5.13) is positive or zero and that the integral is zero if either

(1) $\lambda_{1}^{*} \leq 1$ and $\hat{\lambda}_{1} \leq 1$ throughout $A$, or

(2) $\lambda_{1}^{*} \leq 1$ and $\hat{\lambda}_{1} \leq 1$ in some region $A_{1}$ of $A$ and in the remainder of $A$ we must have that $\lambda_{1}^{*}>1, \hat{\lambda}_{1}=\lambda_{1}^{*}$, and $\hat{\lambda}_{1}^{*}=\hat{\lambda}_{1}\left(\varphi_{1}^{*}=\hat{\varphi}_{1}\right)$.

An identical argument applied to the second integral in (5.13) leads to the conclusion that this integral is positive or zero and that the integral is zero if either

(1) $\lambda_{2}^{*} \leq 1$ and $\hat{\lambda}_{2} \leq 1$ throughout $A$, or

(2) $\lambda_{2}^{*} \leq 1$ and $\hat{\lambda}_{2} \leq 1$ in some region $A_{2}$ of $A$ and in the remainder of $A$ we must have that $\lambda_{2}^{*}>1, \hat{\lambda}_{2}=\lambda_{2}^{*}$, and $\hat{\lambda}_{2}^{*}=\hat{\lambda}_{2}\left(\varphi_{2}^{*}=\hat{\varphi}_{2}\right)$.

We note that the equalities $\hat{\lambda}_{1}=\lambda_{1}^{*}, \hat{\lambda}_{1}^{*}=\hat{\lambda}_{1}$ imply that $\hat{\varphi}_{1}=\varphi_{1}^{*}, \widehat{T}_{11}=T_{11}^{*}$, $\widehat{T}_{12}=T_{12}^{*}$ and that the equalities $\hat{\lambda}_{2}=\lambda_{2}^{*}, \hat{\lambda}_{2}^{*}=\hat{\lambda}_{2}$ imply that $\hat{\varphi}_{2}=\varphi_{2}^{*}, \widehat{T}_{21}=$ $T_{21}^{*}, \widehat{T}_{22}=T_{22}^{*}$. Thus, if the two integrals in equation (5.13) are both zero then the functions $\hat{\lambda}_{\alpha}, \hat{\varphi}_{\alpha}, \hat{x}_{\alpha}, \widehat{T}_{\alpha 1}$, and $\hat{T}_{\alpha 2}$, in addition to satisfying equations (5.3), (5.4), (5.5), also satisfy the equilibrium equations (5.6) and the traction boundary conditions (5.7) and hence constitute a solution of the governing equations. Our results may then be summed up in the following theorem.

THEOREM 1. For networks with strain-energy functions of the form (5.12), a solution of the general boundary-value problem gives a local minimum of the functional (5.1).

By applying this theorem to two different solutions of the same boundary-value problem we are led to conclude that any two solutions must yield the same value of the functional (5.1). Using this with the above results then leads to the generalized uniqueness theorem.

THeOREM 2. Assume that the functions $W_{1}\left(\lambda_{1}\right)$ and $W_{2}\left(\lambda_{2}\right)$ take the form (5.12). 
(1) If the whole region is fully-stretched, then the solution is unique.

(2) If half-slack regions or fully-slack regions exist, then the solution may not be unique, but the fully-stretched region in one solution is fully-stretched in every solution and in this subregion the solution is unique; the half-slack region in one solution is half-slack in every solution and the tensions and directions of the stretched cord lines are unique; the fully-slack region in one solution is fully-slack in every solution.

This is similar to Pipkin's theorem [5] for inextensible networks.

6. Uniqueness and stability with generalized strain-energy functions. Besides their positivity and increasing with the arguments of $W_{1}\left(\lambda_{1}\right)$ and $W_{2}\left(\lambda_{2}\right)$ in (5.2), we assume that they are such that $\widetilde{W}_{\alpha}^{\prime}$ and $\widetilde{W}_{\alpha}^{\prime \prime}$ exist at $\lambda_{\alpha}=1$. If we define

$$
W\left(\lambda_{1}, \lambda_{2}\right)=\widetilde{W}_{1}\left(\lambda_{1}\right)+\widetilde{W}_{2}\left(\lambda_{2}\right)
$$

then from $(5.9)$

$$
\frac{\partial W}{\partial \lambda_{1}}=\frac{d \widetilde{W}_{1}}{d \lambda_{1}}=T_{1}\left(\lambda_{1}\right), \quad \frac{\partial W}{\partial \lambda_{2}}=\frac{d \widetilde{W}_{2}}{d \lambda_{2}}=T_{2}\left(\lambda_{2}\right) .
$$

Substituting these into (5.8) with (2.3) and (2.5) or (5.4), we have

$$
T_{\alpha \beta}=\frac{\partial W}{\partial \lambda_{\alpha}} \frac{1}{\lambda_{\alpha}} \frac{\partial x_{\beta}}{\partial X_{\alpha}}
$$

From continuum mechanics theory (see Ogden [12] and Spencer [13]), we note that both $\lambda_{1}$ and $\lambda_{2}$ are not, in general, the principal stretches, since they are not the eigenvalues of the Green-Lagrange strain tensor. In fact, they are the square roots of the major diagonal components of the Green-Lagrange strain tensor. Then we can see that $W\left(\lambda_{1}, \lambda_{2}\right)$ represents the strain-energy function for the kind of material considered here.

Introducing the components of the displacement vector

$$
u_{1}=x_{1}-X_{1}, \quad u_{2}=x_{2}-X_{2},
$$

we can express the stretches in an alternative way

$$
\lambda_{1}=\left\{\left(u_{1,1}+1\right)^{2}+u_{2,1}^{2}\right\}^{1 / 2}, \quad \lambda_{2}=\left\{u_{1,2}^{2}+\left(u_{2,2}+1\right)^{2}\right\}^{1 / 2}
$$

and then the strain-energy function is

$$
W=\widetilde{W}_{1}\left(\sqrt{\left(u_{1,1}+1\right)^{2}+u_{2,1}^{2}}\right)+\widetilde{W}_{2}\left(\sqrt{u_{1,2}^{2}+\left(u_{2,2}+1\right)^{2}}\right)
$$

where $u_{j, i}=\partial u_{j} / \partial X_{i}$.

Hill [11] has investigated the uniqueness and stability of the elastic deformation with finite strain by using the material description. An equilibrium state is said to be stable if, in the motion following an arbitrary disturbance, the amplitude of the additional displacement is always vanishingly small when the disturbance itself is. He concluded that if the strain-energy function $W$ were a strictly convex function of the displacement gradients, then the solution would be unique and stable (assuming 
that the boundary conditions exclude a superimposed rigid-body movement). Alternatively, if we define

$$
C_{i j k l}=\frac{\partial^{2} W}{\partial\left(u_{j, i}\right) \partial\left(u_{l, k}\right)}, \quad(i, j, k, l=1,2,3),
$$

then the solution would be unique and stable if the quadratic form associated with the tensor $C_{i j k l}$ were positive definite, i.e.,

$$
C_{i j k l} \Delta_{i j} \Delta_{k l}>0 \text { unless all } \Delta_{i j}=0 .
$$

Since the material we are considering is of the strain-energy function (6.5), the associated quadratic form, after some manipulations, is

$$
\begin{aligned}
C_{\alpha \beta \gamma \nu} \Delta_{\alpha \beta} \Delta_{\gamma \nu}= & \frac{\partial^{2} W}{\partial\left(u_{\beta, \alpha}\right) \partial\left(u_{\nu, \gamma}\right)} \Delta_{\alpha \beta} \Delta_{\gamma \nu} \\
= & \frac{\widetilde{W}_{1}^{\prime \prime}}{\lambda_{1}^{2}}\left[\left(u_{1,1}+1\right) \Delta_{11}+u_{2,1} \Delta_{12}\right]^{2}+\frac{\widetilde{W}_{1}^{\prime}}{\lambda_{1}^{3}}\left[\left(u_{1,1}+1\right) \Delta_{12}-u_{2,1} \Delta_{11}\right]^{2} \\
& +\frac{\widetilde{W}_{2}^{\prime \prime}}{\lambda_{2}^{2}}\left[\left(u_{2,2}+1\right) \Delta_{22}+u_{1,2} \Delta_{21}\right]^{2}+\frac{\widetilde{W}_{2}^{\prime}}{\lambda_{2}^{3}}\left[\left(u_{2,2}+1\right) \Delta_{21}-u_{1,2} \Delta_{22}\right]^{2},
\end{aligned}
$$

where $\alpha, \beta, \gamma, \nu=1,2$.

If not all $\Delta_{\alpha \beta}(\alpha, \beta=1,2)$ vanish, then the terms in the brackets are not all zero. This can be shown as the following. If

$$
\begin{aligned}
& \left(u_{1,1}+1\right) \Delta_{11}+u_{2,1} \Delta_{12}=0, \\
& \left(u_{1,1}+1\right) \Delta_{12}-u_{2,1} \Delta_{11}=0, \\
& \left(u_{2,2}+1\right) \Delta_{22}+u_{1,2} \Delta_{21}=0, \\
& \left(u_{2,2}+1\right) \Delta_{21}-u_{1,2} \Delta_{22}=0,
\end{aligned}
$$

then the only solutions are $\Delta_{\alpha \beta}=0(\alpha, \beta=1,2)$ since

$$
\left(u_{1,1}+1\right)^{2}+u_{2,1}^{2}=\lambda_{1}^{2}>0 \text { and }\left(u_{2,2}+1\right)^{2}+u_{1,2}^{2}=\lambda_{2}^{2}>0 .
$$

This result contradicts the assumption that not all $\Delta_{\alpha \beta}$ vanish.

Assume that the cords are such that $\widetilde{W}_{\alpha}^{\prime \prime}>0$ when $\lambda_{\alpha}>1 \quad(\alpha=1,2)$. Then in the fully-stretched region, $\widetilde{W}_{\alpha}^{\prime}=T_{\alpha}\left(\lambda_{\alpha}\right)>0 \quad(\alpha=1,2)$, the quadratic form (6.8) is positive definite and the deformation is unique and stable if there exists a solution to the deformation. But in a half-slack region, $\widetilde{W}_{1}^{\prime}=T_{1}=0$ or $\widetilde{W}_{2}^{\prime}=T_{2}=0$ (also $\widetilde{W}_{1}^{\prime \prime}=0$ or $\widetilde{W}_{2}^{\prime \prime}=0$ ), the quadratic form can be zero while not all $\Delta_{\alpha \beta}$ are zero. In a fully-slack region, $\widetilde{W}_{1}^{\prime}=T_{1}=0$, and $\widetilde{W}_{2}^{\prime}=T_{2}=0 \quad$ (also $\widetilde{W}_{1}^{\prime \prime}=\widetilde{W}_{2}^{\prime \prime}=0$ ), the quadratic form is always zero. So in these regions the deformation may not be unique. Therefore we may conclude the following.

THEOREM 3. If $\widetilde{W}_{\alpha}^{\prime \prime}>0$ when $\lambda_{\alpha}>1 \quad(\alpha=1,2)$, then

(1) if there is a solution to the deformation in which the whole region is fullystretched, then the deformation is unique and stable; 
(2) if there is a solution to the deformation in which fully-stretched, half-slack and/or fully-slack regions exist simultaneously, then there may be other deformations due to the existence of the half-slack and/or fully-slack regions. But a fully-stretched region in one solution is a fully-stretched region in every solution. This is because the deformation in a fully-stretched region is unique and hence this region cannot become a half-slack or fully-slack region.

The results obtained here are for a general form of the strain-energy function which is restricted only to be a sum of two functions of separate arguments and to satisfy the condition $\widetilde{W}_{\alpha}^{\prime \prime}>0$. The quadratic form in Sec. 5 is a special case of those discussed here. The first part of the results is identical with that obtained in Sec. 5, but with the argument used in this section, we cannot prove that a halfslack region in one solution is half-slack in every solution, a fully-slack region in one solution is fully-slack in every solution, and the tensions and directions of stretched cords in half-slack regions are unique. To obtain these last results, if possible, other considerations may be needed.

Aknowledgment. Jingyu Shi is very grateful to the Agricultural Ministry of the People's Republic of China and the ORS Awards Committee of the United Kingdom for finanical support.

\section{REFERENCES}

[1] R. S. Rivlin, Plane strain of a net formed by inextensible cords, J. Rational Mech. Anal. 4. 951-974 (1955)

[2] T. G. Rogers and A. C. Pipkin, Holes in inextensible networks, Quart. J. Mech. Appl. Math. 33, 447-462 (1980)

[3] A. C. Pipkin, Some developments in the theory of inextensible networks, Quart. Appl. Math. 38, 343-355 (1980)

[4] A. C. Pipkin, Plane traction problems for inextensible networks, Quart. J. Mech. Appl. Math. 34, 415-429 (1981)

[5] A. C. Pipkin, Inextensible networks with slack, Quart. Appl. Math. 40, 63-71 (1982)

[6] A. C. Pipkin, Energy minimization for nets with slack, Quart. Appl. Math. 44, 249-253 (1986)

[7] A. C. Pipkin and T. G. Rogers, Infinitesimal plane wrinkling of inextensible networks, J. Elasticity 17, 35-52 (1987)

[8] S. M. Genensky and R. S. Rivlin, Infinitesimal plane strain in a network of elastic cords, Arch. Rational Mech. Anal. 4, 30-44 (1959-60)

[9] W. A. Green and J. Shi, Plane deformations of membranes formed with elastic cords, Quart. J. Mech. Appl. Math. 43, 317-333 (1990)

[10] J. Shi, Elastic networks and membranes, Ph.D. Thesis, Nottingham University, U.K., 1988

[11] R. Hill, On uniqueness and stability in the theory of finite elastic strain, J. Mech. Phys. Solids 5, 229-241 (1957)

[12] R. W. Ogden, Nonlinear elastic deformations, Ellis Horwood Limited, Chichester, 1984

[13] A. J. M. Spencer, Continuum mechanics, Longman, New York, 1980 